\title{
Practising shared decision making in primary care
}

\author{
Ping Yein Lee, Chirk Jenn Ng
}

Lee PY, Ng CJ. Practising shared decision making in primary care. Malays Fam Physician. 2021;16(1);2-7. https://doi.org/10.51866/cm0001

\section{Keywords:}

Shared decision making,

primary care, patient decision

aids, implementation.

\section{Authors:}

$\mathrm{Ng}$ Chirk Jenn

(Corresponding author) MBBS, MMed Family Med, PhD

Department of Primary Care

Medicine, Faculty of Medicine

University of Malaya, Kuala Lumpur

Malaysia

Email: ngcj@um.edu.my

\section{Lee Ping Yein}

MBBS, MMed Family Med

Department of Family Medicine Faculty of Medicine and Health

Sciences, Universiti Putra

Malaysia, Serdang, Malaysia

\section{Abstract}

Making healthcare decisions collaboratively between patients and doctors can be challenging in primary care, as clinical encounters are often short. Conflicts between patients and doctors during the decision-making process may affect both patient and doctor satisfaction and result in medicolegal consequences. With the increasing recognition of the importance of patient empowerment, shared decision making (SDM) can serve as a practical consultation model for primary care doctors (PCDs) to guide patients in making informed healthcare choices. Although more research is needed to find effective ways to implement SDM in the real world, the 6-step approach presented in this paper can guide PCDs to practise SDM in their daily practice. Implementation of SDM can be further enhanced by incorporating SDM training into undergraduate and postgraduate curricula and using evidence-based tools such as patient decision aids.

\section{Box 1. Case Study 1: Making a decision about starting insulin in a patient with type 2 diabetes}

Sally, a 68-year-old woman, has been diagnosed with type 2 diabetes mellitus for the past 15 years. She attends the Health Clinic nearby every 4 months for her diabetes check. Sally was able to achieve good control initially for the first few years; more recently, her doctor had to increase the dosage of her oral medications and add new medications to keep her diabetes under control.

During this clinic visit, Sally meets Dr Jon, a newly graduated family medicine specialist, who advises her to consider starting insulin. Dr Jon explains to Sally that her blood glucose control has deteriorated over the past 1 year (Hbalc : 8-9\%) despite taking three oral medications. Sally is surprised as she has been taking her medications regularly without fail and is extremely careful with her diet and exercise routine. She is not keen to start insulin as she is fearful of needles and pain with injections.

Dr Jon patiently explains to Sally that insulin will lower her blood glucose level and with good control, she is less likely to develop complications such as blindness, kidney failure and heart attacks. Sally is in a dilemma; while she does not like the idea of having diabetes complications (she has seen her mother suffering from kidney failure), she cannot bear the thought of having to inject herself daily. Moreover, she has been living alone since her husband's death 2 years ago, and her only son is living abroad. She is worried that she may have 'hypos' at home.

Issues to consider:

1. Sally is conflicted. She wants the best of both worlds; she wants to avoid diabetes complications but also to avoid the side effects of insulin.

2. Dr Jon is frustrated. He wants to help Sally to manage her diabetes better, and insulin is the obvious choice, but Sally is not accepting his advice.

3. Is there a way that Sally and Dr Jon can come to decision that both of them are happy with?

Box 2. Case study 2: Practising shared decision making with a man who requests prostate cancer screening

Richard, a 70-year-old man, requests his general practitioner, Dr Ian, screen him for prostate cancer. Richard is worried because his friend has just died of prostate cancer.

Dr Ian asks about Richard's risk of getting prostate cancer; fortunately, Richard does not have any family history of prostate cancer. Dr Ian then explains to him about the screening option, 
which is doing a prostate specific antigen (PSA) test. He explains that the other screening option, performing a digital rectal examination, is not as accurate as PSA test. That said, a PSA test is not very accurate as a screening tool; it may lead to 'false positive' (PSA level is high even though Richard does not have prostate cancer) and 'false negative' (PSA level is normal but Richard actually has prostate cancer) result. Richard begins to understand that the PSA test is not foolproof.

Dr Ian goes on to inform Richard about the implication of doing a PSA test. If the PSA test returns as high, Richard might be referred to a urologist for further assessment, where either a biopsy or MRI will be done to find out whether Richard indeed has prostate cancer. Richard asks questions about what a prostate biopsy entails, and Dr Ian explains the procedure, including the potential side effects such as pain, bleeding and infection. In addition, there may be risk of complication of incontinence and erectile dysfunction when prostate cancer is treated with prostatectomy.

Dr Ian then talks about how detecting prostate cancer early can potentially cure the cancer and save lives; however, some prostate cancers are slow-growing and may not need surgery or other treatments. The urologist will have to assess each patient individually and not to treat every patient the same way. Richard understands that the PSA test is not absolutely accurate, and there are consequences to doing it. He also realises that some prostate cancer takes a long time to grow and may not cause death immediately. He now has the choice of whether to go for PSA test, and not every man of his age must go for prostate cancer screening.

After making sure that Richard understands the information, Dr Ian guides Richard to make a decision by finding out what is important to Richard personally: is it the concern of having a prostate cancer, or not wanting to experience the consequences of a high PSA test. Richard values good quality of life and is concern about the complications of prostate biopsy and treatment. Richard decides that he can live with the uncertainty of having a prostate cancer as his risk is not high. He will return if he starts to experience any urinary symptoms so that an early diagnosis can be made. He decides not to have PSA test for now.

\section{Introduction}

Making healthcare decisions collaboratively between patients and doctors can be challenging, especially in primary care, where clinical encounters are often short. ${ }^{1}$ While most healthcare decisions are easy to make, some can be challenging, especially when patients' values differ from those of their doctors, such as whether to initiate insulin (Box 1). On the one hand, patients tend to find it difficult to make a decision as they must bear the consequences, including change in the lifestyle, financial burden and health outcomes. On the other hand, primary care doctors (PCD), who struggle to 'convince' patients to make a decision, may feel frustrated and dissatisfied with the consultation. Conflicts between patients and doctors during the decision-making process may affect both patient and doctor satisfaction and may, in some instances, result in medico-legal consequences. ${ }^{2}$ This warrants a decision-making approach that provides a platform for patients and PCDs to talk openly about their priorities and concerns to reach a consensus on the healthcare decision.
Historically, paternalism dominates healthcare consultations. ${ }^{3}$ Doctors tend to make decisions 'in the best interest of patients', often without involving them in the decision-making process. Clinical paternalism occurs when patients rely heavily on doctors' expertise and experience to decide for them, especially in societies where doctors are highly regarded in the social scale. Patients are also fearful of making a 'wrong' decision that may adversely affect their health; hence, they tend to delegate the decision-making task to the expert (doctor). ${ }^{4}$ However, with an increase in overall literacy and health literacy, and easy access to health information via the Internet, patients are now more empowered to acquire health information independently. ${ }^{5}$ This has shifted the power dynamic between the doctor and patient towards a more balanced partnership.

In primary care, where multiple healthcare decisions are made in each clinical encounter, there is an even more urgent need for PCDs to equip themselves with the skill to guide patients to make informed decisions, especially those with clinical equipoise. ${ }^{6}$ A practical and effective decision-making model, such as SDM, is needed for this to happen. 


\section{Shared Decision Making}

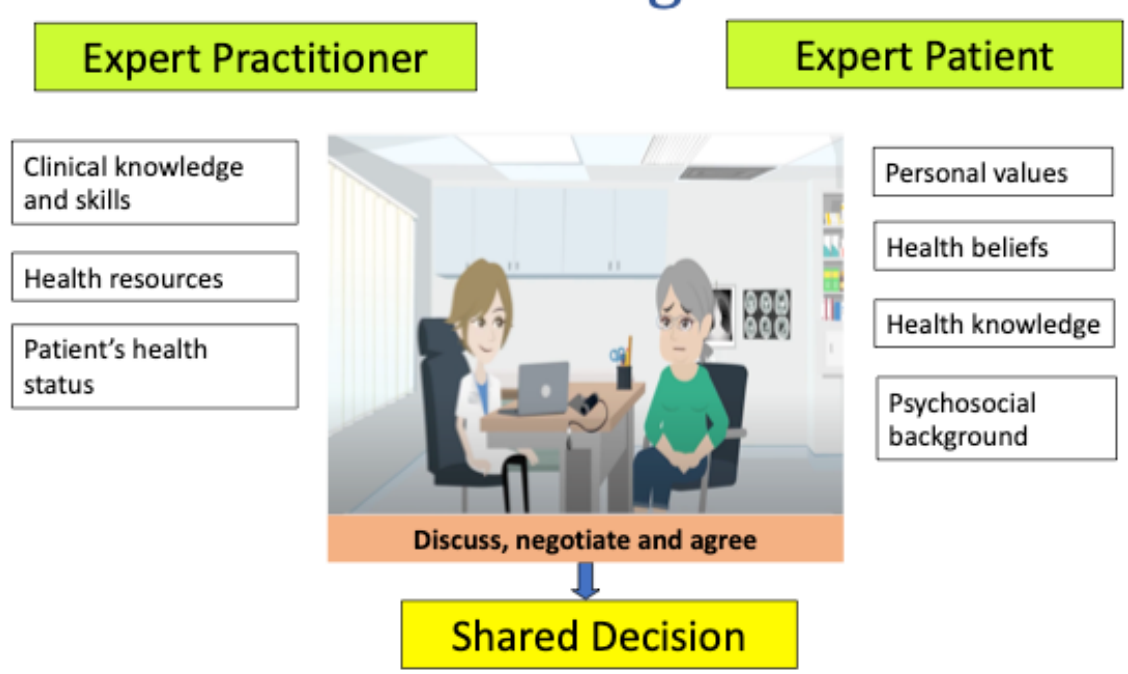

Figure 1: Shared decision-making model $^{7-9}$

\section{Shared Decision Making}

The concept of SDM was developed in the 1980 s out of the need to implement 'informed consent' in the healthcare setting. ${ }^{7}$ A huge practice variation existed within and across healthcare systems; for example, prescribing patterns and uptake of surgical procedures for the same health condition varied significantly. One of the key influencing factors was variation in how healthcare providers made decisions, often independently without patients' involvement and sometimes not following the recommended clinical practice guidelines. While some healthcare decisions are relatively straightforward, when benefits outweigh the risks (e.g. antibiotic use in pneumonia), some decisions are more challenging, especially those with clinical equipoise (e.g. the insulin initiation example described in Box $\mathbf{1}$ and the prostate cancer screening example described in Box 2). SDM requires patients and doctors to communicate their concerns and priorities openly during the clinical encounter.

SDM is a clinical consultation process where a patient and a doctor discuss, negotiate and agree on the final decision. ${ }^{8}$ SDM is usually conducted under the premise that both patients and doctors recognise that both parties' views must be respected. While doctors have clinical expertise and experience, patients are 'experts' in their own values (i.e. the patient knows what is important to them). ${ }^{\text {? }}$ Both patients and doctors have 'equal power' during the decision-making process, which requires building a good rapport between both parties, and a trusting professional relationship is critical. The success of the SDM implementation depends on how well and to what extent 'discussion', 'negotiation' and 'agreement' processes are done. Therefore, SDM requires doctors to embrace patientcentred care, have good communication skills, and recognise the clinical context in which SDM can be used to guide patients in making a decision.

Table 1: Six Steps in Practising Shared Decision Making (SDM)

\begin{tabular}{|l|l|}
\hline Steps & Key Questions \\
\hline 1. Identify the decision & What is the decision? \\
\hline 2. List the available options & What screening, diagnostic or treatment options are available? \\
\hline $\begin{array}{l}\text { 3. Discuss the pros and cons of } \\
\text { each option }\end{array}$ & $\begin{array}{l}\text { What are the advantages and disadvantages of each option? } \\
\text { What is the evidence? }\end{array}$ \\
\hline 4. Elicit patient values & What is important to the patient? \\
\hline 5. Provide support & What further support does the patient need to make this decision? \\
\hline 6. Make a decision & Is the patient ready to make a decision? \\
\hline
\end{tabular}




\section{Step-by-step approach to practising SDM}

Six steps can be used to guide patients to make an informed and value-based decision (Table 1) in SDM. ${ }^{10}$ The following step-by-step approach will use Case Study 2 (Box 2) as an exemplar to demonstrate how SDM can be practised during a primary care consultation.

\section{Step 1: Identify the decision}

It is important to establish what decision needs to be made before discussing SDM with patients. Decisions may be about screening, diagnostic tests or treatment options. In Case Study 1 , the decision is whether to start insulin as a treatment option (other options could be 'status quo' or 'add another medication'); in Case Study 2, the decision is whether to undergo prostate cancer screening by doing a PSA test.

\section{Step 2: List the available options}

After establishing what the decision is, the next step to inform the patient is about the available healthcare options. This step will ensure that the patient is aware of the range of options and that there is no 'right' or 'wrong' option. The final decision depends on how the patient views the options after considering their personal preference and values. In Case Study 2 , the options are to screen or not screen for prostate cancer using the PSA test.

\section{Step 3: Discuss the pros and cons of each option}

A PCD needs to discuss the pros and cons of each of the available options in an unbiased manner. In some instances, the options may include 'do nothing' and 'using complementary and alternative medicine'. All the information should be based on scientific evidence and explained to the patient in simple language.

In Case Study 2, Dr Ian informs Richard about the accuracy of the PSA test (false positives and false negatives) and that a biopsy would be required if the PSA is elevated. In discussing the benefit of screening, Dr Ian explains that early detection of prostate cancer may save lives; however, the doctor can never be sure whether the cancer will be slowgrowing or aggressive. He also talks about the potential harms of screening, including the complications of a prostate biopsy (e.g. bleeding and infection) and prostatectomy (e.g. incontinence and erectile dysfunction).
In communicating the pros and cons of the available options, the PCD should use 'numbers' (e.g. out of 100 patients like you, 20 experience headache) rather than 'percentages' (e.g. 20\%) to present the risks and benefits. ${ }^{11,12,13}$ In addition, to avoid the 'framing effect' (presenting the information positively or negatively to sway decision), it is important to present the chances of getting and not getting complications from the illness or treatment. $^{14}$

\section{Step 4: Elicit patient values}

Identifying the patient's values can be a challenging step in a consultation. ${ }^{10}$ Different individuals have different personal views, concerns and values of what is important to them. Values are an important element that may influence how a patient chooses the treatment or screening option. Patient values often relate to their psychosocial background, health beliefs and health knowledge. Therefore, it is essential to explore patient values before guiding them to make a decision.

In Case Study 2, Richard values good quality of life and is concerned about the complications of prostate biopsy and treatment. He decides not to go for prostate cancer screening as he prioritises the avoidance of the potential consequences of screening over the potential reassurance of a negative prostate cancer screening result.

\section{Step 5: Provide support}

Sometimes, the information provided by the PCD during a consultation may not be adequate. Patients may need more support in terms of health information or family and peer support. Open-ended questions, such as: 'Is there anything else you would like to know?' or 'Is there anyone else you would like to talk to before making a decision?’ may be useful.

\section{Step 6: Make a decision}

The last step is to establish whether the patient is ready to make a decision. Some patients may want to take time to think through the options or discuss with their family or peers before making a decision. In this case, the decision will need to be revisited in the next consultation. PCDs should explore further what other concerns the patient may have and address them accordingly. Patients should not be pressured to make a decision when they are 
not ready. PCDs must accept the possibility of 'no decision' when guiding the patient in making decisions during a consultation.

\section{Using Patient Decision Aids in SDM}

Patient decision aids (PDAs) are evidencebased tools used to help people make informed choices about treatment, diagnostic or screening options. ${ }^{15}$ PDAs provide information about the health condition and decision, list available options, present evidence of the benefits and risks of each option and explore patient values and preferences. PDAs are used as a tool in the SDM process to facilitate active discussion between patients and healthcare providers, leading them to informed decisions. ${ }^{15}$ The use of PDAs must consider the patient's social and cultural context, such as language, family involvement, and use of complementary and alternative medicine. ${ }^{16}$

A Cochrane systematic review confirmed the benefits of using a PDA in healthcare decision making; ${ }^{17}$ it increases patients' knowledge about treatment choices and their pros and cons. With increased knowledge, patients are more empowered to make an informed decision. They are also more satisfied with the treatment decision made and increase their treatment adherence. Using PDAs helps health care providers enhance their ability to provide information to patients and facilitate the decision-making process during a consultation. PDA use has a positive effect on patient-doctor communication, increases job satisfaction and reduces emotional exhaustion. Patients who have used a PDA before a consultation are more likely to be equipped with the necessary knowledge to make a decision and more aware of their concerns and values, ${ }^{17}$ hence allowing healthcare providers to focus on discussing what is relevant to the patient.

Several PDAs have been developed for Malaysian patients. These PDAs include, among others, 1) insulin initiation for type 2 diabetes, 2) early breast cancer treatment, 3) metastatic breast cancer treatment, 4) early prostate cancer treatment, 5) bladder management for spinal cord injury patients. These PDAs are written in different languages (English, Malays, Mandarin and Tamil) to ensure that they are tailored for patients of different ethnicities.

\section{Implementation of SDM in clinical practice}

Despite the increasing evidence to support the use of SDM in clinical practice, implementing SDM and PDAs remain a challenge, particularly in developing countries such as Malaysia, where the concepts of SDM and PDAs are still unfamiliar to policy makers, healthcare providers and patients. ${ }^{18}$ The main challenge is the lack of SDM culture. Doctors' paternalistic attitude, patients' passivity and lack of (or too much) trust in doctors make implementing SDM challenging. ${ }^{19}$ In addition, distinct role boundaries between the doctors and nurses are also a potential barrier. In many primary care clinics, doctors would not expect nurses to be able to deliver PDA information to patients. Furthermore, the lack of continuity of care makes it difficult for doctors to follow up on PDA use with their patients. ${ }^{19}$

To facilitate the implementation of SDM and PDAs, doctors need to be trained on how to practice SDM with their patients and use tools such as PDAs to facilitate decision making during a consultation. ${ }^{19}$ Doctors' communication style and commitment to patient-centred care are likely to influence the implementation outcomes of PDAs. ${ }^{20}$ While time is the most commonly cited barrier to practice SDM, ${ }^{21}$ PDAs may reduce cumulative consultation time as patients are less likely to delay a decision. In addition, a reminder network among the healthcare providers may be useful to address the issue of forgetfulness and trigger interest in using the PDA to encourage SDM. ${ }^{19}$ Health care authorities should create a conducive environment and provide patients with free access to PDAs to promote effective implementation of SDM. ${ }^{20}$

\section{Conclusion}

With the increasing recognition of the importance of patient empowerment, SDM can serve as a practical consultation model for PCDs to guide patients in making informed healthcare choices. Although more research is needed to find effective ways to implement SDM in the real world, the 6-step approach presented in this paper can guide a PCD to begin practising SDM in their daily practice. This practice can be further enhanced by strategies such as including incorporating SDM training into undergraduate and postgraduate training, using evidence-based tools such as PDAs and delivering those tools using technology. ${ }^{18}$ 


\section{References}

1. Irving G, Neves AL, Dambha-Miller H, et al. International variations in primary care physician consultation time: a systematic review of 67 countries. BMJ Open. 2017;7(10):e017902. doi:10.1136/ bmjopen-2017-017902

2. Elwyn G, Edwards A, Kinnersley P. Shared decision-making in primary care: the neglected second half of the consultation. Br J Gen Pract. 1999;49(443):477-482.

3. Sm G. Paternalism in healthcare decision making. Ostomy Wound Manage. 1998;44(4):22, 24-25.

4. Say R, Murtagh M, Thomson R. Patients' preference for involvement in medical decision making: A narrative review. Patient Educ Couns. 2006;60(2):102-114. doi:10.1016/j. pec. 2005.02 .003

5. Aydın GÖ, Kaya N, Turan N. The role of health literacy in access to online health information. Procedia - Soc Behav Sci. 2015;195:1683-1687. doi:10.1016/j. sbspro.2015.06.252

6. Elwyn G, Edwards A, Kinnersley P, Grol R. Shared decision making and the concept of equipoise: the competences of involving patients in healthcare choices. Br J Gen Pract. 2000;50(460):892-899.

7. President's Commission. President's Commission for the Study of Ethical Problems in Medicine and Biomedical and Behavioral Research. Making health care decisions. The ethical and legal implications of informed consent in the patientpractitioner relationship. Washington DC: 1982. Accessed February 18, 2021. https:// repository.library.georgetown.edu/bitstream/ handle/10822/559354/making_health_care_ decisions.pdf
8. Charles C, Gafni A, Whelan T. Shared decision-making in the medical encounter: what does it mean? (or it takes at least two to tango). Soc Sci Med. 1997;44(5):681-692. doi:10.1016/S0277-9536(96)00221-3

9. Murray E, Charles C, Gafni A. Shared decision-making in primary care: tailoring the Charles et al. model to fit the context of general practice. Patient Educ Couns. 2006;62(2):205-211. doi:10.1016/j. pec. 2005.07 .003

10. Schrager SB, Phillips G, Burnside E. A simple approach to shared decision making in cancer screening. Fam Pract Manag. 2017;24(3):5-10.

11. Fagerlin A, Zikmund-Fisher BJ, Ubel PA. Helping patients decide: ten steps to better risk communication. JNCI J Natl Cancer Inst. 2011;103(19):1436-1443. doi:10.1093/jnci/ djr318

12. Zipkin DA, Umscheid CA, Keating NL, et al. Evidence-based risk communication: a systematic review. Ann Intern Med. 2014;161(4):270-280. doi:10.7326/M140295

13. Sinayev A, Peters E, Tusler M, Fraenkel L. Presenting numeric information with percentages and descriptive risk labels: a randomized trial. Med Decis Mak Int J Soc Med Decis Mak. 2015;35(8):937-947. doi:10.1177/0272989X15584922

14. Joseph-Williams N, Newcombe R, Politi $\mathrm{M}$, et al. Toward minimum standards for certifying patient decision aids: a modified Delphi consensus process. Med Decis Mak Int J Soc Med Decis Mak. 2014;34(6):699-710. doi:10.1177/0272989X13501721
15. Patient Decision Aids - Ottawa Hospital Research Institute. Accessed February 17, 2021. https://decisionaid.ohri.ca/

16. Chenel V, Mortenson WB, Guay M, Jutai JW, Auger C. Cultural adaptation and validation of patient decision aids: a scoping review. Patient Prefer Adherence. 2018;12:321-332. doi:10.2147/PPA.S151833

17. Stacey D, Légaré $\mathrm{F}$, Lewis $\mathrm{K}$, et al. Decision aids for people facing health treatment or screening decisions. Cochrane Database Syst Rev. 2017;4:CD001431. doi:10.1002/14651858.CD001431.pub5

18. Ng CJ, Lee PY, Lee YK, et al. An overview of patient involvement in healthcare decisionmaking: a situational analysis of the Malaysian context. BMC Health Serv Res. 2013;13:408. doi:10.1186/1472-6963-13-408

19. Tong WT, Lee YK, Ng CJ, Lee PY. Factors influencing implementation of a patient decision aid in a developing country: an exploratory study. Implement Sci. 2017;12(1):40. doi:10.1186/s13012-0170569-9

20. Tong WT, Ng CJ, Lee YK, Lee PY. What will make patients use a patient decision aid? A qualitative study on patients' perspectives on implementation barriers and facilitators. J Eval Clin Pract. 2020;26(3):755-764. doi:10.1111/ jep. 13161

21. Légaré F, Ratté S, Gravel K, Graham ID. Barriers and facilitators to implementing shared decision-making in clinical practice: update of a systematic review of health professionals' perceptions. Patient Educ Couns. 2008;73(3):526-535. doi:10.1016/j. pec. 2008.07 .018 